\title{
Spectroscopy of pionic atoms with unstable nuclei
}

\author{
Kenta Itahashi ${ }^{a}$ \\ ${ }^{a}$ Advanced Meson Science Laboratory, RIKEN, Japan
}

\begin{abstract}
We are planning an experiment to perform spectroscopy of pionic atoms with unstable nuclei (RI) by using the $d\left(R I,{ }^{3} \mathrm{He}\right) R I^{*} \otimes \pi^{-}$nuclear reaction. The experiment is to be performed at the SIS-100 NESR facility with the incident RI beam energy of $250 \mathrm{MeV} / \mathrm{u}$.
\end{abstract}

\section{Introduction and physics goals}

We have deduced the density dependent part of the strong interaction between a pion and a nucleus [1]. Also, the data are analyzed from the viewpoint of pion mass modification and chiral symmetry restoration of the "vacuum" in the nucleus, which is treated as zerotemperature high density matter stable in the laboratory. We have established evidence of the symmetry restoration and the magnitude of the restoration is estimated.

So far, pionic atom study works as a very powerful tool. However, there is still a strong boundary. The target nuclei must be $\beta$-stable. Here, we propose a further advanced method of nuclear reaction spectroscopy by using the inverse kinematics as described below. Pionic states are sensitive to the structure of nuclear surface, and its spectroscopy will serve an opportunity to investigate the property of unstable nuclei using the pion as a sensitive probe. Figure 1 shows the calculated binding energy and the width of pionictin atom changing the neutron density distribution parameters, radial $(c)$ and diffuseness (a) parameters. Precise determination of pionic states will provide information on the properties of unstable nuclei. In the inverse kinematics reactions, the emitted ${ }^{3} \mathrm{He}$ will have small kinetic energy of about $60 \mathrm{MeV}$. This will help to achieve higher resolution.

There are reasons we propose the experiment to the new facility in the GSI. The new facility will provide the world highest intensity beam of unstable nuclei. The produced beam will be stored in the storage rings. Since the proposed experiment requires high luminosity and small emittance beam, any other facilities in the world do not fulfill the requirement. Hereafter, we will investigate the experimental feasibility of pionic atom spectroscopy with tin-132 nucleus. 


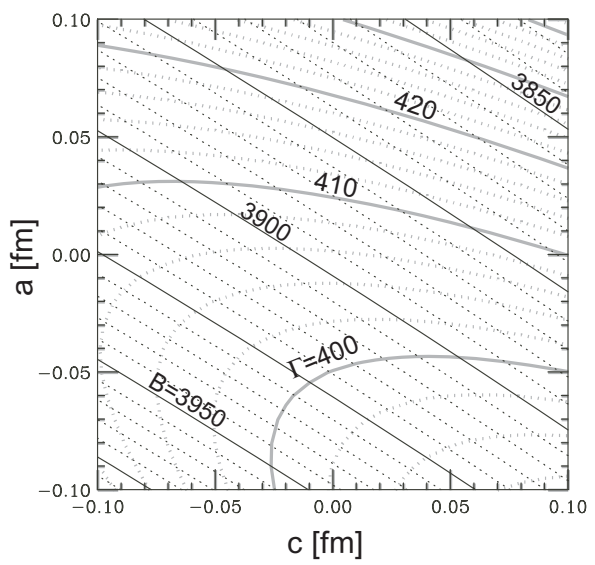

Figure 1: Calculated binding energy and width of pionic tin atom $[\mathrm{keV}]$ by changing the neutron density distribution parameters. The distribution used in the calculation is a two parameter Fermi model, $\rho(r)=\rho_{0} /\left(1+\exp \left[\frac{r-c}{a}\right]\right)$.

\section{Experimental principles}

In the present spectroscopy of pionic atoms, the pion is produced in a reaction and is bound to a nucleus. The elementary reaction is $n+d \rightarrow{ }^{3} \mathrm{He}+\pi^{-}$, thus a neutron is separated from the nucleus simultaneously. The absolute coupling strengths with the neutron states depend on the magnitude of the overlap between the pion and the neutron wave functions. We measure the $Q$-value of the reaction $(Q)$ near the $\pi^{-}$emission threshold and the binding energy $\left(B_{\pi}\right)$ is deduced according to

$$
Q=m_{\pi^{-}} c^{2}-B_{\pi}+S_{n}-\left(M_{d}+M_{n}-M_{3_{\mathrm{He}}}\right) c^{2}
$$

where $S_{n}$ is the neutron separation energy, $m_{\pi^{-}}, M_{d}, M_{n}, M_{3_{\mathrm{He}}}$ respectively show the mass of pion, deuteron, neutron, and ${ }^{3} \mathrm{He}$. We expect to measure the bound state as a peak in the $Q$-value spectrum.

Since the reaction in the center-of-mass frame is the same for the inverse kinematics and the normal kinematics, some of the previous experiences are still effective in the present experiment. The pionic atom formation cross section for the present experiment is calculated by Umemoto et al. [7] and the ambiguity in the calculation is known to be small.

In order to enhance the formation probability of pionic atoms and to suppress the quasi-free pion production, the momentum transfer of the reaction should be small. We have to choose the incident beam energy to ensure the recoilless kinematical condition. Figure 2(left) depicts the momentum transfer dependency on the incident beam energy in case of $d\left(\mathrm{Sn},{ }^{3} \mathrm{He}\right)$ reaction for pionic atom formation. The incident beam energy of $\sim 250$ $\mathrm{MeV} / \mathrm{u}$ is naturally selected for this purpose. 

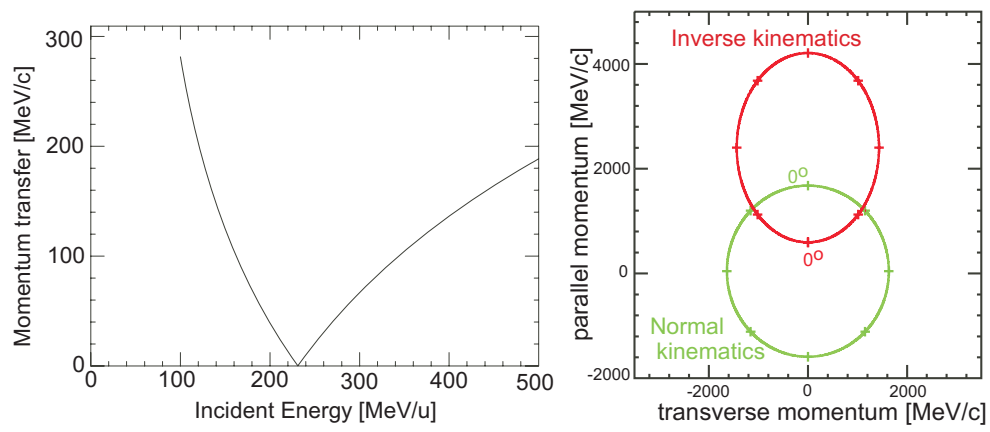

Figure 2: (left)Recoil momentum vs. incident energy in case of $d\left(\mathrm{Sn},{ }^{3} \mathrm{He}\right)$ reaction. Recoilless kenematical condition for pionic atom formation is fulfilled near 250 $\mathrm{MeV} / \mathrm{u}$ incident energy. (right)A $Q$-value contour in the plane of parallel and transverse momenta in the laboratory frame.

A different property between the two kinematics appears in the ${ }^{3} \mathrm{He}$ emission angle dependency of the $Q$-value. Figure 2(right) shows a contour for a certain $Q$-value with both kinematics in the plane of parallel and transverse momentum in the laboratory frame. Emission angle in the center-of-mass frame (CM) is shown by small ticks for $0, \pm 45, \pm 90, \pm 135,180$ degrees. While $Q$-value in the normal kinematics does not depend strongly on the ${ }^{3} \mathrm{He}$ emission angle, in the inverse kinematics the $Q$-value depends on both the kinetic energy of the ${ }^{3} \mathrm{He}$ and the emission angle. Thus, we need to measure both of them to achieve high resolution.

\section{Experimental setup}

In order to take the advantages of the inverse kinematics, the present experiment needs to be performed at a storge ring. Here, to design the experiment in a realistic way, we have taken into account the specifications of the new facilities, SIS-100 and NESR. The tin-133 beam of $250 \mathrm{MeV} / \mathrm{u}$ is produced at the primary target at the entrance of the Super-FRS facility by either projectile fragmentation or fission reaction and separated from other nuclei. The produced tin-133 beam is guided to the NESR facility and the momentum spread is reduced by the cooling system. The beam intensity in the NESR by one injection is assumed to be $\sim 5 \times 10^{7}$ and for the higher yield, we also consider multiple injection to increase the intensity. As for the target, our design is based on the deuteron cluster jet target with the density of $5 \times 10^{14} / \mathrm{cm}^{2}$.

Figure 3 shows the present design of the experimental apparatus. We measure the $Q$-value by measuring the emission angle and the kinetic energy of the ${ }^{3} \mathrm{He}$. Since the formation cross section has a peak in the forward 0 degree, the detector system was designed to have its acceptance accordingly. In the reaction of $d\left({ }^{133} \mathrm{Sn},{ }^{3} \mathrm{He}\right){ }^{132} \mathrm{Sn} \otimes \pi^{-}$, the emitted ${ }^{3} \mathrm{He}$ has kinetic energy of about $60 \mathrm{MeV}$ and is corresponding to about six 
times smaller rigidity than that of the in-ring beam. The ${ }^{3} \mathrm{He}$ is first separated from the beam by the deflection magnet (MD1). The deflection of the beam is compensated by two magnets (MC1 and MC2).

We place the main detectors of a silicon strip detector (SSD) and a planer Germanium detector at about one meter from the target. The SSD is $300 \mu \mathrm{m}$ thick and has $3 \times 3 \mathrm{~cm}^{2}$ effective area with $32+32$ strips in both sides. The emission angle of the ${ }^{3} \mathrm{He}$ is measured as the position on the SSD. The position resolution is about $1 \mathrm{~mm}$ and is sufficient. The energy loss in the SSD is also measured. The total energy of the ${ }^{3} \mathrm{He}$ is measured by $5 \mathrm{~mm}$ thick Germanium detector.

Small amount of material causes deterioration of the resolution because of the energy straggling. Thus, to have the best resolution, the detector system should be located in a vacuum connected to the ultra high vacuum of the storage ring without any separating foil. We are developing a SSD and the Germanium detectors to be operated in such high vacuum. Table 1 summarizes the estimated $Q$-value resolution and the contributions. The estimated resolution is $250 \mathrm{keV}$ (FWHM). The largest contribution is the resolution of the Germanium detector and others are very small.

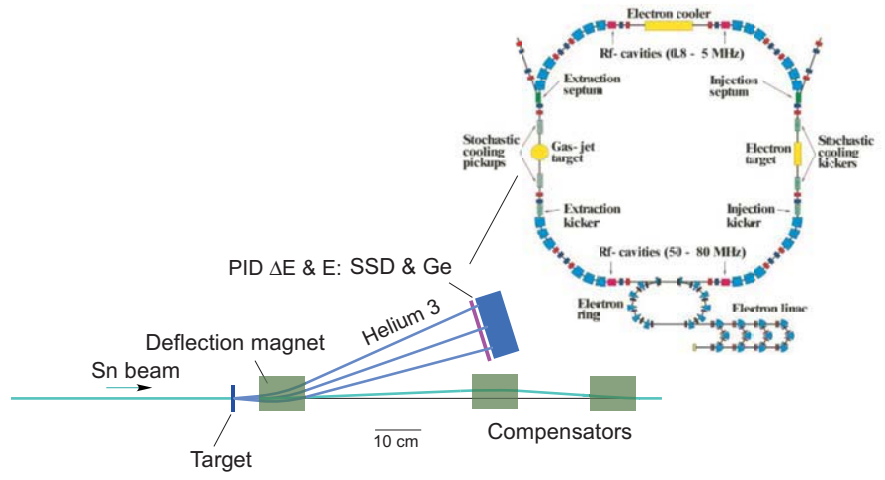

Figure 3: Experimental setup in the NESR.

\begin{tabular}{ll}
\hline Angular resolution & $12 \mathrm{keV}$ \\
Energy resolution of SSD & $75 \mathrm{keV}$ \\
Energy loss straggling in the dead layer of SSD & $50 \mathrm{keV}$ \\
Germanium detector energy resolution & $225 \mathrm{keV}$ \\
\hline$Q$-value resolution & $240 \mathrm{keV}$ (FWHM) \\
\hline \hline
\end{tabular}

Table 1: Energy resolution 


\section{Yield estimation}

The experimental yield is estimated and tabulated in Table 2 .

\begin{tabular}{lc}
\hline Beam & ${ }^{133} \mathrm{Sn} 250 \mathrm{MeV} / \mathrm{u}$ \\
Beam Production Intensity & $1 \times 10^{8} / \mathrm{sec}$ \\
Circulation frequency & $2 \mathrm{MHz}$ \\
Effective Life time & $92 \mathrm{sec}$ \\
Repitition & $10 \mathrm{sec}$ \\
Cross section & $25 \mu \mathrm{barn} / \mathrm{sr}$ \\
Deuteron cluster jet Target & $5 \times 10^{14} / \mathrm{cm}^{2}$ \\
Detector solid angle & $900 \mu \mathrm{Sr}$ \\
\hline Yield & $\sim 20 /$ hour \\
\hline \hline
\end{tabular}

Table 2: Yield estimation.

\section{Summary}

As described briefly, the spectroscopy of pionic atom with unstable nuclei becomes feasible in the newly build facility at the GSI. This experiment needs elaborate R\&D process to brush up all experimental apparatus to achieve higher resolution and higher statistics. The work is in progress.

\section{References}

[1] K. Suzuki et al., Phys. Rev. Lett. 92, 72302 (2004).

[2] W. Weise, Nucl. Phys. A 690, 98 (2001).

[3] Y. Akaishi and T. Yamazaki, Phys. Rev. C 65, 044005 (2002).

[4] M. Iwasaki, et al., nucl-ex 0310018 (2003).

[5] T. Yamazaki et al., Z. Phys. A 355, 219 (1996).

[6] H. Geissel et al., Phys. Rev. Lett. 88, 122301 (2002).

[7] Y. Umemoto et al., Nucl. Phys. A 679 (2001) 549. 
